\title{
L'exercice selon Laurent Chiflet (1659). Spiritualité et pédagogie jésuite
}

The exercise according to Laurent Chiflet (1659). Jesuit spirituality and pedagogy

\section{Cendrine Pagani-Naudet}

\section{(2) OpenEdition \\ 12 Journals}

Édition électronique

URL : https://journals.openedition.org/dhfles/6722

DOI : $10.4000 /$ dhfles. 6722

ISSN : 2221-4038

Éditeur

Société Internationale pour l'Histoire du Français Langue Étrangère ou Seconde

Édition imprimée

Date de publication : 1 décembre 2019

Pagination : 141-165

ISBN : 0992-7654

ISSN : 0992-7654

Référence électronique

Cendrine Pagani-Naudet, "L'exercice selon Laurent Chiflet (1659). Spiritualité et pédagogie jésuite », Documents pour l'histoire du français langue étrangère ou seconde [En ligne], 62-63 | 2019, mis en ligne le 27 avril 2020, consulté le 28 mars 2023. URL : http://journals.openedition.org/dhfles/6722 ; DOI : https://doi.org/10.4000/dhfles.6722

Ce document a été généré automatiquement le 28 mars 2023.

Tous droits réservés 


\title{
L'exercice selon Laurent Chiflet (1659). Spiritualité et pédagogie jésuite
}

The exercise according to Laurent Chiflet (1659). Jesuit spirituality and pedagogy

\author{
Cendrine Pagani-Naudet
}

1 Existe-t-il un rapport entre une pratique méditative ancienne, appelée exercices spirituels, et la pratique pédagogique, objet du présent colloque ? Il est difficile d'éluder la question quand on travaille sur une grammaire jésuite, en l'occurrence l'Essay d'une parfaite grammaire de Laurent Chiflet, paru à Anvers en $1659^{1}$. Cet ouvrage, dans lequel Joseph Hanse reconnaît «la contribution belge à la définition du bon usage », s'inscrit dans la double continuité des grammaires françaises ${ }^{2}$ et des Remarques ${ }^{3}$. Composé, au moins en partie, pour répondre à la demande d'un lectorat flamand, c'est aussi une grammaire pour étrangers. C'est même une méthode de langue ${ }^{4}$. Plus précisément, cette grammaire peut se transformer en méthode de langue : c'est une relecture que propose la section intitulée "La manière d'enseigner et d'apprendre la langue française » (Chiflet 1659: 151-155) ${ }^{5}$ et que rend possible l'organisation matérielle de l'ouvrage. Ce dernier est outillé de manière à servir plusieurs types d'usagers : « ceux qui sçavent desja la Langue, \& n'ont besoin que de s'y perfectionner : les autres qui en veulent apprendre les Principes, tels que sont les étrangers " (Chiflet 1659, Préface NP). Le livre a donc cette plasticité, cette adaptabilité si caractéristique des Jésuites, « dextérité sociale » (Lacouture 1991 : 106) dont les manifestations sont politiques aussi bien que pédagogiques. L'Essay est en effet une grammaire jésuite, et la section sur « la manière d'enseigner et d'apprendre » est une proposition pédagogique jésuite. Chiflet y expose une progression fondée sur l'exercice, sur la mise en activité de l'apprenant, activité encadrée, orientée, répétable et graduée, obéissant à une progression qui n'est pas sans rappeler le parcours que décrit Ignace de Loyola dans les Exercices spirituels, texte fondateur de la Compagnie. 


\section{L'exercice dans la section sur « La manière d'apprendre et d'enseigner »}

\section{Articulation de la section à la grammaire}

2 Parmi les usagers potentiels du livre, Chiflet envisage le maître de langue auquel sont plus particulièrement destinées les pages consacrées à «La manière d'apprendre et d'enseigner » qui forment la section 7 du chapitre 8 « De la syntaxe et du style ${ }^{6}$. Cette section propose une progression pour un non francophone. La référence à l'apprenant flamand est récurrente. Non seulement dans la section sur « la manière d'enseigner » :

Par exemple, pour les Flamands. J'ay, ick hebbe : tu as, ghy hebt : il a, hy heeft. (Chiflet

$1659: 152)$

Quant aux Allemands et aux Flamands, il faut avoir un grand soing de leur apprendre l'usage de l'Optatif. (Chiflet $1659: 153$ )

mais aussi plus explicitement dans la section non numérotée, qui lui fait suite: $l^{\prime}$ «Adjonction particulière pour les flamands $»^{7}$. Chiflet y dresse la liste des fautes les plus courantes chez l'apprenant flamand. Il n'est pas inutile de rappeler que la même année paraît une version flamande abrégée de la grammaire de Chiflet, dans laquelle on retrouve l'essentiel de la morphologie verbale et la liste des flandricismes ${ }^{8}$.

4 La découverte de la langue pour un non francophone ne suit pas l'ordre de la grammaire tel qu'il est présenté dans la table des matières : décrire le système de la langue et l'enseigner sont deux choses distinctes. Pour le débutant, il convient de commencer par le verbe. C'est une recommandation présente dès début de l'ouvrage : Chiflet explique qu'il a déplacé l'exposé sur la prononciation pour permettre à l'apprenant de " parcourir au plutost les plus necessaires principes de nostre langue, \& arriver en moins de temps aux Conjugaisons des Verbes, qui sont, à dire le vray, comme l'ame de tout le langage, \& qu'il faut apprendre plus soigneusement que tout le reste » (Chiflet 1659: 6). Néanmoins, la prononciation reste la première chose que le maître doit enseigner à son élève. La préconisation est claire : «Il faut avant toute chose, que le Maistre enseigne à son disciple la façon de bien prononcer, en lisant avec luy " (Chiflet 1659 : 151). Il revient donc au maître de recomposer à partir du livre imprimé un autre livre, de sélectionner point par point ce qui convient à son élève à chaque étape de sa formation. La conception matérielle du livre rend cette circulation possible. L'Essay comporte en effet une véritable table des matières (et non un index) détaillée et paginée, avec une hiérarchisation fine des titres. Un système de numérotation des sections et des alinéas permet au lecteur par des renvois internes une circulation, à la fois souple et orientée ${ }^{9}$. Dès le seuil de l'ouvrage, la préface suggère la possibilité d'une telle lecture, en procédant à des renvois internes :

Vous en aurez de claires preuves, dans la Seconde Partie de cette Grammaire, au no. 24. de la septieme Section du Premier Traité ; \& au no. 2. du Troisieme Traité » (Chiflet 1659, préface NP).

Outil auquel Chiflet a naturellement recours pour guider le maître :

[...] \& à cet effet leur faire apprendre les Regles de l'yfage de l'optatif, qui font en cette Grammaire, au Chap. 4. fect. 8. n. 4 \& au Chap. 7. sect. 2. n. 3. (Chiflet 1659 : 153). 


\section{Un enseignement gradué}

6 L'auteur insiste sur le caractère gradué de la méthode. Chaque étape est signifiée par l'emploi d'un adverbe :

Le lendemain, qu'il fasse reïterer la mesme lecture (Chiflet 1659 : 153)

Puis avec d'autres Substantifs, qui tirent aprés eux des Verbes infinitifs (...). Puis ceux cy: J'ay besoin d'argent, tu as faute de courage, Il a soin de mes affaires. En suite, vous ferez conjuguer le mesme Verbe auec des Noms accompagnez d'articles (Chiflet $1659: 153$ ).

Aprés cet auxiliaire, il faut apprendre l'autre [...] Puis y ajouter un Nom [...] En suite, qu'il apprenne un Verbe Regulier de la premiere conjugaison, dont l'Infinitif est terminé en er: comme, Aimer. [...] Enfin de la quatrieme en re: comme Rendre, vendre, Respondre. (Chiflet $1659:$ 154)

7 Chiflet conseille une découverte progressive de la langue, qui permettra à l'élève de s'exercer dans des domaines de compétence variés: d'abord, la lecture, la prononciation, l'écriture, l'orthographe ${ }^{10}$, la récitation, et la manipulation syntaxique sur des énoncés brefs; puis la traduction, la narration, et la conversation ${ }^{11}$. L'organisation de la section suggère quatre ou cinq étapes. La première est consacrée à l'apprentissage simultané de la prononciation (à travers la lecture à voix haute et la récitation des leçons) et de l'étude du verbe. Outre la morphologie, la mémorisation des conjugaisons donne déjà des rudiments de syntaxe et du lexique.

8 La mise en place des rudiments nécessitant des précautions importantes, cette première étape fait l'objet d'une description détaillée ${ }^{12}$. Elle occupe près de deux pages, et Chiflet en résume l'esprit avant de passer à la deuxième étape :

Donc en ces premiers jours, il y aura tousjours exercice de la lecture, et de dire par cœur les conjugaisons apprises, y ajoutant, comme j'ay dit, quelques mots de leur construction avec des Noms ou avec d'autres Verbes. (Chiflet 1659 : 154)

Chiflet y expose un modèle de progression, que le maître pourra étendre ensuite de manière analogique :

Au premier jour, il faut aussi donner par escrit le Verbe auxiliaire J'ay, tu as, il a, ou elle $a$ etc. [...]. Quand le Disciple aura bien appris tout ce Verbe, il lui faut donner divers exemples du mesme Verbe, avec les noms accusatifs, qu'il a coustume de regir sans articles. Exemple. J'ay faim, tu as soif, il a peur. Nous avons etc. J'ay droit, tu as tort, il a raison. Nous avons etc. J'ay chaud, tu as froid, il a mal aux dents. Nous avons etc. [...] Puis avec d'autres Substantifs, qui tirent aprés eux des Verbes infinitifs: comme : J'ay envie de rire, tu as besoin d'apprendre, il a raison de se plaindre. Puis ceux cy : J'ay besoin d'argent, tu as faute de courage, Il a soin de mes affaires. En suite, vous ferez conjuguer le mesme Verbe avec des Noms accompagnez d'articles: comme, J'ay vn bon pere; th as un beau miroir, il a une belle maison. J'ay la colique, tu as mon argent, il a de beaux habits. (Chiflet 1659 : 152-153).

10 À partir du verbe avoir, l'élève est amené à mémoriser des séquences de plus en plus complexes, passant de locutions comme «j'ai faim » ou «j'ai peur » à des combinaisons de plus en plus libres ( il a de beaux habits»). Au-delà de la conjugaison, le disciple découvre les parties du discours: nom, article, adjectif, infinitif, préposition. Cette première étape contribue à l'enrichissement progressif du lexique (à travers des couples d'antonymes «faim »/ «soif », « droit »/ «tort », « chaud»/ «froid»), et met en place des routines de communication, des formules associées à des situations simples. 
11 La même méthode est étendue aux autres verbes: au verbe substantif, aux verbes réguliers, aux « principaux Verbes irreguliers » (Chiflet 1659 : 153). Chiflet recommande de «tousjours ajouter à chaque Verbe quelques noms, aprés qu'on les aura appris tous seuls » (Chiflet 1659 : 153). Ces paradigmes enrichis donnent immédiatement accès aux idiotismes, aux collocations, à des ensembles lexicaux complexes, mémorisés avant que n'en soient abordées les règles combinatoires: cette méthode permet de fixer des patrons syntaxiques sans faire appel à des connaissances métalinguistiques. Les règles seront explicitées plus tard, dans la troisième phase de l'apprentissage.

12 Apprendre la langue par principes n'aurait d'ailleurs pas permis de réaliser toutes ces séquences idiomatiques. Que Chiflet propose de commencer par là indique une conscience assez nette de ce qui pose problème dans l'acquisition d'une langue. La question des locutions, des expressions conventionnelles et inversement des calques est abordée sous les deux angles dans deux sections consécutives : dans «la manière d'apprendre", la mémorisation des paradigmes verbaux est aussi l'occasion d'apprendre les locutions les plus courantes; inversement, "l'Adjonction pour les flamands » met en garde contre les flandricismes. D'une section à l'autre, certains exemples se répondent:

Puis ceux cy : J'ay besoin d'argent, tu as faute de courage, Il a soin de mes affaires.

(Chiflet 1659: 152).

42. J'ay besoin un peu d'argent. M'avez vous affaire. Dites, J'ay besoin d'un peu

d'argent. Avez vous affaire de moy? (Chiflet $1659: 163$ ).

y ajoutant des adjectifs; un chapeau gris, un manteau rouge \&c. (Chiflet $1659: 154)$.

46. Un gris chapeau, un rouge manteau \&c. Le langage Flamand met tousjours

l'adjectif devant le Substantif. Dites, chapeau gris, Manteau rouge \&c. (Chiflet 1659 :

163).

13 Chiflet trouve un compromis entre l'apprentissage par principes et l'apprentissage par routine : le disciple apprend d'abord par cœur des séquences, ce qui le sensibilise à des principes dont la formulation est toutefois différée.

14 «Les conjugaisons étant bien apprises »- l'expression marque bien l'importance de ce prérequis permettant le passage à l'étape suivante -, le maître intègre les autres parties du discours (nom, pronoms, indéclinables). C'est en réalité un enrichissement et un approfondissement de ce qui a été appris durant les premiers jours. On arrive ensuite à une troisième étape : «Il ne restera plus que de donner quelques regles generales de la Syntaxe» (Chiflet 1659: 154). En réalité, comme dans les autres chapitres de la grammaire ${ }^{13}$ il s'agit d'expliciter des principes qui ont été expérimentés au moment de l'apprentissage des parties du discours. En outre, le maître intègre à cette occasion les rudiments de la conversation civile. La quatrième étape introduit de nouveaux exercices: il s'agit désormais d'unifier les apprentissages à travers des activités de traduction et de conversation :

Tout le reste de l'instruction consistera à luy faire traduire quelque chose du latin ou de quelque autre langue, en François : et à parler avec lui, le corrigeant de ce qu'il dit ou prononce mal. (Chiflet 1659: 155).

La dernière étape est celle de la consolidation :

Aprés qu'il aura bien appris, il faut qu'il conserve ce qu'il sçait, en lisant tous les jours dans quelque bon livre. (Chiflet 1659 : 155).

16 Le disciple entretient et perfectionne sa connaissance de la langue, par la lecture critique des bons auteurs, des bons livres, par la réflexion métalinguistique, par l'exercice de la parole en continu (faire raconter quelques histoires). 


\section{Un enseignement dynamique} compléter : sont donc conjointement impliqués le maitre et le disciple. La démarche de Chiflet consiste non pas à faire une leçon, puis à faire appliquer, mais plutôt à mettre immédiatement l'élève en situation de pratiquer : à partir d'éléments de langue que l'élève lit, écrit, récite, seront donnés des éléments de correction d'abord, puis, progressivement, des explications. C'est un apprentissage dynamique qui procède par enrichissements successifs (à chaque étape, le maître "ajoute »), et qui vise à unifier progressivement les éléments de langue appris. Par l'entraînement, le travail sur soi progressif et régulier, l'élève est conduit à la maîtrise complète de la langue, c'est-àdire à une forme de liberté dans la pratique. La mise en place d'une routine permet de déclencher la formation d'énoncés nouveaux, de l'énoncé figé à l'énoncé librement composé : les paradigmes, les dialogues appris par cœur deviennent une matière dynamique. L'apprentissage est un processus envisagé dans sa durée: on perçoit la progression de l'élève à travers la complexification des tâches qui lui sont proposées.

\section{Retour aux sources}

\section{Pédagogues et maîtres de langue}

Le bon sens pédagogique que manifeste cette section n'étonne pas de la part de Laurent Chiflet, souvent qualifié de "praticien $»^{15}$. La section sur "la manière d'enseigner " peut d'ailleurs se lire comme un héritage, une synthèse, plus ou moins consciente, d'influences diverses. Chiflet s'inscrit dans la tradition pédagogique humaniste. L'idée d'un enseignement gradué et sollicitant régulièrement le disciple est déjà présente chez Érasme. Dans son plan d'étude ${ }^{16}$, ce dernier insiste sur l'importance de l'entraînement, impliquant l'exercice de la mémoire, les exercices de lecture et d'explication d'auteurs ${ }^{17}$. Ces principes sont à l'œuvre dans un texte dont l'intitulé n'est pas sans rappeler celui adopté par Chiflet : La Maniere d'exercer les enfans a decliner les noms et les verbes $^{18}$, paru chez Robert Estienne et attribué à Mathurin Cordier. On y retrouve le même souci de la prononciation qui doit se faire "clerement et entendiblement " (Cordier 1546 : 162), l'importance accordée au support écrit, à la récitation, le rôle de la répétition d'une même procédure ${ }^{19}$. Difficile également d'ignorer l'influence possible de Comenius, en dépit des oppositions que l'on peut concevoir entre ces deux univers ${ }^{20}$. La toute nouvelle méthode des langues (Novissima linguarum methodus) parue en 1648 énonce des axiomes qui semblent également - quoique non explicités - au cœur de « la manière d'enseigner " exposée par Chiflet. Comenius ainsi conseille un enseignement gradué ${ }^{21}$, 
insiste sur le rôle du maitre ${ }^{22}$, sur le recours à la langue de l'apprenant, sur l'importance de la pratique intelligemment articulée à l'exposition des règles ${ }^{23}$.

En matière d'enseignement du français aux étrangers, Chiflet n'invente rien non plus. Depuis le début du XVIe siècle, il existe une production abondante d'ouvrages destinés à l'apprentissage du français pour les étrangers. Le territoire des Pays-Bas et d'Anvers ${ }^{24}$ est particulièrement fertile: les besoins en maîtres de langue y sont anciens et le XVIe siècle a connu une importante floraison de grammaires pratiques, dialogues, et dictionnaires. Certains de ces ouvrages sont toujours imprimés du temps de Chiflet. Ainsi à côté d'auteurs comme La Grue, ou d'Arsy, il faut encore compter avec Berlaimont ou Meurier ${ }^{25}$. Chez tous ces auteurs, Chiflet a pu trouver le souci de maintenir le lien avec la langue maternelle: vocabulaires et dialogues sont souvent bilingues, voire plurilingues ${ }^{26}$. Il leur emprunte aussi la démarche consistant à faire "manipuler des ensembles structurés avant de les décomposer en constituants" (Boone 2000 : 335). Comme eux, il privilégie d'abord la mémorisation de séquences complexes avant d'introduire les explications et les concepts métalinguistiques. Articuler aux conjugaisons des séquences linguistiques complètes rappelle ainsi les exemples « par formes de parler» de Meurier ${ }^{27}$.

\section{La manière d'enseigner à la lumière des Exercices spirituels}

Héritier de la pensée pédagogique humaniste, Chiflet reste en outre conforme à l'esprit de sa congrégation. On pense aux principes formulés dans le Ratio Studiorum, mais ce texte est lui-même fortement inspiré des Exercices spirituels de Loyola. Charmot souligne la " parenté très étroite qui existe entre les principes des Exercices de Saint Ignace et les principes de pédagogie du Ratio " (Charmot 1943: 137). Et J.-Y. Calvez le rappelle : " pour le Ratio, tout ou presque est exercice : s'exercer soi-même, exercer autrui ; ceci est en rapport direct avec les Exercices spirituels (Calvez 2001: 15). La section sur «la manière d'enseigner et d'apprendre » illustre cette continuité entre la pédagogie et la spiritualité jésuites, entre le Ratio studiorum et les Exercices spirituels. Pourquoi cependant remonter du Ratio à sa source, aux Exercices spirituels? C'est que, à l'époque de Chiflet, l'enseignement du français échappe encore à l'institution, et se déroule en marge du curriculum que décrit le Ratio. Et c'est précisément pourquoi dans la "Manière d'enseigner ", les conditions d'apprentissage semblent plus proches de ce que Loyola décrit dans les Exercices spirituels que des règles qui rythment la vie des collèges.

Les Exercices spirituels (dont la première édition latine paraît en 1548) correspondent à une œuvre vivante au sein de la compagnie de Jésus, comme pratique, et comme produit éditorial: on ne compte plus les rééditions, traductions ${ }^{28}$, et autres produits dérivés: livres de piété, parfois intitulés exercices. Ces succédanés n'étaient pas d'ailleurs sans pervertir quelque peu l'esprit même de l'ouvrage fondateur, mais le rendaient de ce fait plus conforme à l'esprit de la Contre-Réforme ${ }^{29}$. Laurent Chiflet, prêtre et membre de la compagnie, devait lui-même sa renommée éditoriale à ses ouvrages pieux, parmi lesquels figurent des exercices spirituels ${ }^{30}$. Aussi même dans ses rares emplois le terme exercice devait-il être chargé de sens, pour celui dont Jean Boyvin dans sa chronique sur le siège de Dôle rappelle qu'il «fut l'inventeur et le directeur de presque tous les vœux et devots exercices que l'on a prattiqués dans la ville» (Boyvin 1637: 118). Chiflet semble avoir étendu au français, discipline dont 
l'enseignement n'était pas encore défini et encadré par l'institution, son expérience de pédagogue et de directeur spirituel.

\section{La relation pédagogique}

23 Les Exercices spirituels proposent, selon un programme de quatre semaines, des exercices de méditation et de contemplation destinés à aider à la prise de décision, à trouver la volonté de Dieu. Les exercices sont précédés de 18 annotations ${ }^{31}$, conseils et mises en garde adressés à celui qui donne les exercices ${ }^{32}$. Le cadre ainsi défini rappelle la relation maitre/élève envisagée par Chiflet. Ce dernier ne décrit pas une situation de classe, la relation est de nature binaire, comme la relation directeur de conscience/ exercitant. Chiflet inaugure "la manière d'apprendre et d'enseigner " par de fermes recommandations adressées au maître de langue :

Il faut avant toute chose, que le Maistre enseigne à son disciple la façon de bien prononcer, en lisant avec luy. [...] Au premier jour, il faut aussi donner par escrit le Verbe auxilaire J'ay, tu as, il a, ou elle a \&c. afin que le disciple ait quelque chose à apprendre par cœur, à la maison, attendant le retour du Maistre. (Chiflet 1659 : 151-152).

La relation d'apprentissage est un enseignement particulier, privé : disciple s'emploie au singulier, on parle de la "maison ». Cela renvoie à la situation du français et de son enseignement à cette époque : il n'était pas enseigné dans les collèges, mais plutôt dispensé « au logis ». Par la relation ainsi définie - un maître, un disciple - Chiflet se distingue de Comenius, qui pense son enseignement pour un groupe, " plusieurs élèves plutôt qu'un seul» (Comenius [1648] 2005: 283), ou de Mathurin Cordier chez qui l'exercice peut devenir collectif (récitation distribuée et répartie, un élève récite le fragment d'une série, un autre enchaîne).

Dans les Exercices Spirituels, la personnalité du directeur spirituel ne doit pas faire écran entre Dieu et celui qui fait les exercices. Il donne les points principaux, à partir desquels il revient à l'exercitant de faire son propre travail. Cette recommandation figure dans la $2 \mathrm{e}$ annotation :

2. La seconde est, Que celuy qui donne à un autre la maniere, \& l'ordre de mediter ou de contempler, luy doit exposer fidellement la matiere de la Meditation ou Contemplation, en parcourant seulement les principaux points legerement, \& y adjoutant une courte explication, afin que celuy qui doit mediter, ayant oüy le veritable fondement de l'histoire, discoure dessus \& forme luy-mesme ses raisonnemens. Car il arrive ainsi qu'ayant trouvé par son propre travail, ou receu par la lumiere divine quelque eclaircissement, ou une plus vive apprehension de l'histoire ; il y prendra plus de plaisir \& il en tirera plus de fruit qu'il feroit si la chose luy eust été déclarée plus au long par un autre. Car ce ne sont pas les grandes connoissances mais les sentimens, \& le goût interieur des choses, qui a coûtume de contenter le desir de l'ame ${ }^{33}$. (Loyola $1673: 31-32$ ).

Dans "la manière d'enseigner ", Chiflet suggère une attitude analogue. On peut interpréter comme une recommandation de réserve et de discrétion les nombreux rappels à la nécessité de s'en tenir à quelques éléments. Le maître ne doit donner "qu'une demi-page», "peu de », «quelques» (mots, enseignements), "grossièrement». Cette "pédagogie de réserve de la part de l'enseignant, une pédagogie incitant le jeune homme ou la jeune femme à agir par soi-même, l'incitant à intérioriser les choses, à ne pas se laisser écraser par la matière ou les contenus, à être attentif à son affectivité, est clairement une pédagogie jésuite » (Calvez 2001: 25). La 
réserve du maître est une des conditions d'une pédagogie active, elle permet à celui qui apprend de mettre en œuvre ses capacités.

Le maître reste en retrait, mais c'est bien lui qui dirige et qui adapte le livre aux besoins de son élève. Le livre est écrit pour le maître. La grammaire telle qu'elle est envisagée dans la section "Manière d'enseigner » est destinée au maître, comme les Exercices Spirituels sont destinés à celui qui donne les exercices, « à l'accompagnateur et non pas directement à celui qui fait l'expérience " (Demoustier 2006 : 12). Le texte des Exercices Spirituels est une « matrice destinée à être transmise en étant accommodée, adaptée en fonction de ceux à qui on la destine " (Demoustier 2006 : 13). Cet esprit de proportion, essentielle dans les Exercices Spirituels et caractéristique de l'esprit jésuite, est rappelé à plusieurs reprises dans l'ouvrage :

La septiéme est, Que si celuy qui conduit un autre dans les Exercices remarque qu'il soit dans la desolation, ou dans la tentation, il doit bien prendre garde à ne pas se montrer facheux et severe envers luy ; mais plutôt facile et doux, en l'affermissant dans la resolution d'agir desormais courageusement; et en luy decouvrant les articifices de l'ennemy pour le disposer à la consolation, qui ne tardera gueres à venir. (1678: 33-34).

La dix-huitième annotation: Qu'il faut accommoder les Exercices à celuy qui les fait, ayant égard à son âge, à sa capacité, \& à son naturel ; de peur de charger un homme grossier, foible, et infirme de ce qu'il ne peut pas porter commodement, et avec fruit. Pareillement selon la fin que chacun se propose, il faut luy donner ce qui peut l'y ayder plus considerablement. C'est pourquoy a celuy qui n'a dessein que de se faire instruire, et d'arriver à quelque degré de vertu où il puisse avoir le repos de son ame, on pourra donner l'Examen particulier mis en la page 45, puis le general page $65^{34}$ [...]. Semblablement si le Directeur en voit un autre qui soit de foible complexion, et de peu de capacité, ce qui fait qu'on n'en puisse gueres esperer d'avancement \& de fruit, ce sera mieux fait de luy ordonner quelques-uns des Exercices plus aisez jusqu'à la Confession des pechez. (Loyola 1678 : 37-38).

Cet usage du livre rapproche Chiflet de Loyola et le distingue de pédagogues comme Mathurin Cordier, pour qui le livre constitue le modèle à suivre: le maitre avance «selon l'ordre du livre» (Cordier $1546: 169$ ), il fait écrire à l'élève « en ung papier la leceon qu'il luy aura faicte, tout ainsi qu'il la trouvera imprimée dans le livre » (Cordier 1546 : 164). L'effort de l'élève porte ainsi sur la leçon (y compris le métalangage) autant que sur la matière linguistique. Ce que le jésuite ajoute à cette manière d'exercer les enfants, c'est un usage plus souple du livre, adaptant le parcours à l'élève. Le maître s'adapte aussi en recourant quand il le faut à la langue maternelle, et ce tant que l'élève en a besoin. Il ajuste son enseignement aux besoins spécifiques liés aux caractéristiques de la langue maternelle : une attention particulière est ainsi accordée à l'optatif pour les Allemands et les Flamands.

Ce rapport au maitre et au livre est une condition de cette pédagogie proportionnée, qui permet à l'élève de progresser.

\section{La répétition}

Aux yeux d'un pédagogue, la répétition est un principe assez banal : l'élève apprend, récite, recommence. Chiflet semble toutefois y ajouter la dimension jésuite de la répétition: un peu plus qu'une simple réitération, c'est un retour critique sur l'expérience. Dans les Exercices spirituels, on passe d'un exercice à l'autre par répétition :

Le quatrieme exercice se fait par repetition du troisiéme. Cette repetition $d u$ troisiesme exercice se met comme une reprise des choses que l'on à méditées dans 
les Exercices precedens, pour les considerer, \& gouter de nouveau, afin qu'en ayant un souvenir continüel, l'entendement raisonne sur elles sans estre exposé aux distractions. Et il faudra ajoûter les trois mesmes Colloques. (Loyola 1673 : 67). partir du point sur lequel on revient, chaque retour est une consolidation et un dépassement. Cette démarche est manifeste chez Chiflet. La progression décrite autour le verbe avoir illustre ce cheminement: la récitation de la conjugaison conduit à une reprise sans cesse enrichie, de plus en plus complexe et de plus en plus libre. Le maître interroge, vérifie les acquis et apporte des explications toujours plus précises. Il parle avec l'élève, «le corrigeant de ce qu'il dit ou prononce mal », plus tard, il l'invite à «faire des réflexions » sur ses lectures. Le travail conjoint de l'élève et du maitre se rapproche du retour sur l'expérience, si caractéristique des exercices spirituels : ces derniers « disposent à une expérience, permettant de la relire, d'en prendre conscience et d'en parler, parole qui rend possible l'exercice suivant pour à nouveau disposer de l'expérience» (Demoustier 2006: 180). Ce qui revient finalement, transposé dans le domaine des apprentissages, à respecter le rythme de l'apprenant, et à s'assurer de l'acquisition des fondements avant d'aller plus loin. C'est bien la démarche de Chiflet, que l'on pourrait qualifier de "spiralaire", tout comme les exercices auxquels Demoustier applique la métaphore : « le point de départ se présente comme une spirale, ressort dessiné à plat. La pratique des exercices en libèrera progressivement la force dynamique et le transformera en spirale » (Demoustier 2006:56).

\section{La progression : un « chemin vers la perfection »} Chiflet aux quatre semaines du parcours spirituel? L'apprentissage de la langue est sans doute étranger aux notions mystiques d'élection, d'abandon de soi pour se fondre en Dieu mais il y a bien un peu de ça tout de même: il s'agit d'oublier la langue maternelle, pour accéder à l'autre langue, de manière intuitive et spontanée. Aussi la progression et le rythme du texte rappellent-ils ceux des Exercices Spirituels. Une accélération se produit, qui montre que quelque chose d'essentiel, d'exponentiel se produit à un moment donné qui permet d'accéder à une nouvelle dimension de l'apprentissage.

Le parcours proposé par les Exercices spirituels est décrit dans la quatrième annotation :

la premiere semaine s'employe à la consideration des pechez: la seconde à la Meditation de la vie de Nostre Seigneur, jusqu'à son entrée en Jerusalem, le jour des Rameaux : la troisiéme à sa Passion : \& la quatriéme à sa Resurrection, \& à son Ascension. (Loyola $1673: 32$ ).

Le terme semaine n'est pas à prendre au sens strict. Chaque étape a sa durée propre, selon le rythme de chacun. Il existe en outre des sortes de pallier. Il en va ainsi du passage de la première à la deuxième semaine. Cette première semaine peut être assez longue car elle suppose une forme de «conversion» (Demoustier 2006: 136) : il faut éradiquer la faute, se libérer de son ancienne vie, s'amender de ses péchés. Certaines âmes grossières n'iront pas au-delà de cette première étape. Une autre étape importante est marquée par l'élection à l'issue de la deuxième semaine. Après les exercices, le chemin n'est pas fini. L'édition parisienne des Vrais exercices s'achève ainsi sur cette recommandation, « pour conserver le bien spirituel acquis par les Exercices »:

Documents pour l'histoire du français langue étrangère ou seconde, 62-63 | 2019 
Tiercement il le faut exciter a conserver et entretenir la devotion jà conceüe par de pieux et spirituels Exercices, entre lesquels ceux cy sont fort recommandables. Premierement qu'il continue l'usage de mediter tous les jours une demie ou l'heure entiere, si faire se peut. Secondement, qu'il fasse tous les jours un quart d'heure d'Examen de conscience. Tiercement qu'il se confesse et communie tous les huict jours. Bref qu'il s'efforce d'acquerir toute la perfection qu'il pourra selon son estat, et la mesure de la grace divine. (Loyola $1623: 411-412$ ).

Le texte de Chiflet, dans sa structure et dans son esprit, ne semble pas étranger à cette progression, à cette tension vers la perfection. Même si le parcours n'a rien à voir avec l'ascétisme, l'apprenant étranger doit pourtant bien opérer une sorte de conversion qui consiste à abandonner progressivement sa langue maternelle. Au début de l'apprentissage, Chiflet conseille de recourir à la langue maternelle, mais seulement tant que cela est nécessaire. Parce qu'il importe aussi dès le seuil d'éradiquer les fautes, Chiflet a consacré une section à les recenser. Et quand il dénonce les errements où mènent les mauvais maîtres, c'est du « mal » et de la « corruption » qu'il s'agit:

En ce point, le mal des Flamands est qu'ils ont souvent de mauvais Maistres en nostre langue. Car il vient aux Pays Bas, plusieurs François naturels, qui ne sçavent que la langue corrompue du commun peuple de leurs Provinces, \& font d'estranges fautes en parlant. (Chiflet $1659: 156$ ).

L'apprenant, conscient de ses erreurs, doit pouvoir s'en défaire, pour entrer dans la nouvelle langue, se laisser habiter par un nouveau système linguistique. La progression décrite par Chiflet est marquée par des seuils, inscrits dans le texte. Il y a une première étape, assez longue, qui consiste à la découverte graduée des parties du discours et au lexique des échanges courants. Ce premier seuil est marqué par le passage à la syntaxe :

Il ne restera plus que de donner quelques regles generales de la Syntaxe ou construction des parties du discours. (Chiflet 1659 : 154).

A partir de là, on peut tendre à la perfection ou s'en tenir à une pratique courante, celle dont on a besoin dans sa vie quotidienne. La syntaxe est le moment où se fait le départ. Les rudiments sont acquis, il est possible de s'en tenir là ou de poursuivre. La lecture critique des bons livres et des bons auteurs arrive en dernier comme couronnement de l'apprentissage et comme manière d'entretenir les connaissances acquises. C'est là qu'intervient l'idée de perfection, terme qui dans le domaine spirituel renvoie «à celui qui a accompli tous les exercices, achevé son cheminement. Le parfait est celui qui est allé au bout de sa formation, cela ne signifie pas l'absence de défaut mais une formation poussée à son terme, pas une signification morale mais pédagogique " (Demoustier 2006 : 73). Chiflet achève la section en recommandant une lecture assidue des textes en vue d'un perfectionnement :

Aprés qu'il aura bien appris, il faut qu'il conserve ce qu'il sçait, en lisant tous les jours dans quelque bon liure. Mais afin que cette lecture lui soit fort utile, il faudra l'enseigner à faire des reflexions sur, l'usage des regles qu'il aura apprises. Par exemple, quand il rencontrera des Optatifs, lui en faire remarquer l'usage. Et ainsi des autres regles de la Grammaire, que le Maistre doit sçavoir parfaitement, afin de monstrer au disciple comment il y faut faire reflexion, en lisant les bons autheurs. Cette façon de lecture est le plus court chemin à la perfection de la langue. Il ne restera plus que de faire raconter quelques histoires au Disciple, pour l'accoutumer à parler promptement \& correctement. (Chiflet 1659 : 155).

L'exercice ne cesse pas : il est différent mais il est permanent. Il repose essentiellement sur un art de lire, lequel rappelle ce qui est préconisé dans la quatrième semaine :

Afin d'imprimer plus fortement dans son esprit les trois contemplations qu'on aura faites ce jour-là : en remarquant aussi en passant et considerant avec plus 
d'application les parties et endroits où nous avons eu les mouvemens plus forts, et des gouts spirituels plus grands. (Loyola $1678: 155$ ).

\section{Conclusion}

Dans une histoire de l'exercice, quelle place accorder à Chiflet? Sa "manière d'enseigner et d'apprendre la langue française » - dont le titre annonce le De ratione discendi et docendi de Jouvency - est remarquable par sa clairvoyance. Une sorte d'évidence s'en dégage, tout semble y relever du bon sens. C'est que ce texte est d'abord le fruit d'un siècle de réflexion pédagogique et d'enseignement des langues. Comme tel, il vaut au moins pour la qualité du témoignage qu'il nous livre sur l'exercice à son époque. Peut-on lui concéder une quelconque influence? Au moins celle qui passe par le réseau des collèges jésuites. La méthode de Chiflet, si peu dépaysante après tout, est profondément marquée par l'esprit de sa Compagnie, et semble même revenir aux origines. La relation pédagogique qu'il envisage renoue en effet avec l'authenticité du texte fondateur très largement perverti au fil du temps. Cette méthode de langue de Chiflet doit ainsi autant à l'expérience du directeur de conscience, qu'à celle du maître de langue ${ }^{35}$. Et si l'on admet avec Jean Stéfanini la modernité de cette «manière d'enseigner ${ }^{36}$, c'est la permanence d'un modèle qu'il faut alors considérer, celle de la pédagogie jésuite, enracinant jusqu'à nos jours son influence.

\section{BIBLIOGRAPHIE}

\section{Sources primaires}

BERLAIMONT, Noël (de) (1511). Vocabulaire pour apprendre à bien lire, escrire et parler françoys et flameng. Anvers : Guillaume Vorstermann.

BOYvin, Jean (1637). Le Siège de la ville de Dôle. Dôle : Antoine Binart.

CHIFLET, Laurent (1659). Essay d'une parfaite Grammaire de la langue françoise. Anvers : Jacques Van Meurs.

CHIFLET, Laurent (1659). Inleydinghe tot de francoysche taele. Anvers : Jacques Van Meurs.

COMENIUS, Jan Amos ([1648] 2005). La toute nouvelle méthode des langues. Methodus Linguarum Novissima. Traduit du latin par Honoré Jean avec l'aide de Gilles Bibeau, Jean Antoine Caravolas et Claire Le Brun-Gouanvic. Genève : Droz.

CORDIER, Mathurin (1546). La Maniere d'exercer les enfans a decliner les noms et les verbes. Paris : Robert Estienne.

LOYOLA, Ignace de (1628). Les vrais exercices spirituels. Paris : Jean Fouet.

LOYOLA, Ignace de (1635). Exercia spirtualia s. p. Ignatii Loyolae. Antverpiae : Joannem Meursius. 
LOYOLA, Ignace de (1673). Les Exercices spirituels de S. Ignace de Loyola. Anvers : Michel Cnobbaert. MEURIER, Gabriel (1557). La Grammaire françoise, contenante plusieurs belles reigles propres \& necessaires pour ceulx qui desirent apprendre ladicte langue. Anvers : Plantin.

\section{Sources secondaires}

BERRÉ, Michel \& BESSE, Henri (2012). « Méthodes, techniques d'enseignement du français comme L2 : éléments pour une réflexion historiographique ». Recherches et Applications - Le Français dans le Monde, 52, 62-77.

BERRÉ, Michel \& PAGANI-NAUDET, Cendrine (2018). « Les flandricismes. Histoire de la grammaire et histoire de la langue ». In B. Colombat, B. Combettes, V. Raby et G. SIoufFI (dir.). Histoire des langues et histoire des représentations linguistiques. Paris : Champion, 501-518.

BOONE, Annie (2000). « Le Paralléle de la grammaire des deux langues françoise et flamende de JacquesFrançois van Geesdalle « 1669) ». In J. De Clerq, N. Lioce et P. Swiggers. Grammaire et enseignement $d u$ français, 1500-1700. Paris/Leuven : Peeters, 335-349.

BOURQUIN, Jacques (2003). Galerie des linguistes franc-comtois. Besançon : Presses universitaires franc-comtoises.

CAlvez, Jean-Yves (2001). « Le Ratio, charte de la pédagogie des jésuites ». Études, 395/9, 207-218. CARAVOLAS, Jean Antoine (1984). Le Gutenberg de la didacographie, ou, Comenius et l'enseignement des langues. Montréal : Guérin.

CARAVOLAS, Jean Antoine (1994). La didactique des langues. Précis d'histoire I. 1450-1700. Montréal : Presses Universitaires de Montréal ; Tübingen : Narr Verlag.

CARAVOLAS, Jean Antoine (2000). «Johann Theodor Jablonski (1654-1731) et la Parfaite grammaire royale de Des Pepliers ». In J. De Clerq, N. Lioce et P. Swiggers. Grammaire et enseignement du français, 1500-1700. Paris/Leuven : Peeters, 463-482.

CHARMOT, François (1943). La pédagogie des jésuites. Paris : Spes.

CLAES, Frans (2000). « Vocabulaires et livres de conversation pour apprendre le français aux PaysBas espagnols entre 1550 et 1700 ». In J. De Clerq, N. Lioce, P. Swiggers. Grammaire et enseignement du français, 1500-1700. Paris/Leuven : Peeters. 217-236.

DE CLERCQ, Jan (2000). « La Grammaire françoise (1557) de Gabriel Meurier ». In J. De Clerq, N. Lioce, P. Swiggers. Grammaire et enseignement du français, 1500-1700. Paris/Leuven : Peeters, 237-276.

DEMOUSTIER, Adrien (2006). Les Exercices spirituels de S. Ignace de Loyola. Lecture et pratique d'un texte. Paris : Éditions des facultés jésuites de Paris.

HANSE Joseph (1962). « La contribution belge à la définition du 'Bon usage' ». Cahiers de l'Association internationale des études françaises, 14, 25-37.

LACOUTURE, Jean (1991). Jésuites. Vol. 1. Les Conquérants. Paris : Seuil.

MARGOLIN, Jean-Claude \& MICHEL, Alain (1976). « Un maître ouvrage de pédagogie humaniste : le plan des études d'Érasme (1512) ». Bulletin de l'Association Guillaume Budé, 3, 273-275.

MONGINI, Guido. « Le discernement dans les Exercices spirituels d'Ignace de Loyola ». Théologiques, volume 22, numéro 2, 2014, 99-135. 
PAGANI-NAUDET, Cendrine (2016). « Le 'bel ordre' de Laurent Chiflet. Espace graphique et description linguistique ». Dossiers d'HEL (Histoire, Épistémologie, Langage), Écriture(s) et représentations du langage et des langues, 9, 163-179. En ligne : [https://hal.archives-ouvertes.fr/hal-01304894].

PELLANDRA, Carla (1990). « L'enseignement du français dans quelques collèges de jésuites du $\mathrm{XVII}^{\mathrm{e}}$ siècle au XVIII ${ }^{\mathrm{e}}$ siècle ». Documents pour l'Histoire du français langue étrangère ou seconde, 6 , 73-81.

RocheMONTEIX, Camille de. Un collège jésuite au XVII et XVIII siècle. Le Mans : Leguicheux, 1889.

STÉFANINI, Jean (1954). « La tradition grammaticale et les temps surcomposés ». Annales de la Faculté des lettres d'Aix-en-Provence, XXVIII, 67-108.

STÉFANINI, Jean (1994). Histoire de la grammaire. Textes réunis par Véronique Xatard. Paris : CNRS Éditions.

SWIGGERS, Pierre (2013). « Regards sur l'histoire de l'enseignement du français aux Pays-Bas (XVI - XVII ${ }^{e}$ siècle) ». Documents pour l'histoire du français langue étrangère ou seconde, 50, 49-79.

VRÉGILLE, Bernard de. « Laurent Chifflet, Jésuite Bibliographie, 1635-1659 ». In Vrégille, Des imprimeurs aux mystiques. Mélanges de bibliographie religieuse comtoise : XVI ${ }^{e}-\mathrm{XVII}{ }^{e}$ siècles. Textes réunis par Manuel Tramaux. Besançon : Cêtre, 2011c, 162-173.

\section{NOTES}

1. L'ouvrage a connu de nombreuses rééditions: à Anvers chez Van Meurs (1664, 1681), à Bruxelles chez Pierre Vleugart $(1675,1683,1688)$ et Lambert Marchant $(1680,1692,1697)$, à Mons chez Gaspard Migeot (1675), à Cologne chez Pierre Legrand (1680), à Paris sous le titre Nouvelle et Parfaite Grammaire de la langue française, chez la Veuve de Louis Gontier (1691), chez Pierre Ribou $(1700,1710,1722)$. L'ouvrage passe pour avoir été la grammaire de référence dans les collèges de la Compagnie, du moins en France, avant d'être détrôné par la Grammaire sur un plan nouveau de Claude Buffier (cf. De Rochemmonteix 1889).

2. Chiflet connait bien la tradition grammaticale française. Ses inventaires se fondent souvent sur les listes établies par Oudin (1632), qui a lui-même actualisé la grammaire de Maupas (1607). C'est sans doute Oudin qu'il faut reconnaître derrière « un certain Grammairien, qui a composé une Grammaire assez bonne, \& la meilleure de toutes celles que j'ay pû voir » (Chiflet 1659: 243). Chiflet connait également la grammaire de Claude Irson, qu'il désigne explicitement dans sa discussion sur recouvert/recouvré, (Chiflet 1659: 94), il a sous les yeux l'édition de 1656 de sa Nouvelle methode pour apprendre facilement les principes et la pureté de la langue françoise dont il donne la pagination : « je n'ay pû m'empescher de rire, en lisant ces paroles, dans la Grammaire de Claude Irson, page 107 ». Irson a sans doute inspiré Chiflet dans l'organisation sa grammaire (intégration des remarques de Vaugelas, section sur la manière d'écrire les lettres, et surtout le chapitre sur la syntaxe et le style).

3. Vaugelas est une référence permanente : Chiflet y puise un nombre important d'exemples et de règles, qu'il discute au besoin. Il rend compte également de la position adverse, la " censure » de La Mothe Le Vayer et Dupleix.

4. L'approbation de 5 décembre 1658 insiste sur cette dimension de l'ouvrage: «Cest Essay parfaite Grammaire de la Langue Françoise par le R. P. LAURENT CHIFLET de la Compagnie de JESUS contient et enseigne une facile methode pour exactement parler et ortographer le François » (Chiflet 1659, Approbation). De fait, la grammaire expose une méthode, à savoir un ensemble de règles et de principes facilitant l'apprentissage progressif de la langue. C'est l'objet de la section «De la manière d'enseigner et d'apprendre » qui montre comment recomposer l'Essay, pour en 
faire une méthode, un manuel délivrant le matériel pédagogique ordonné pour cet enseignement.

5. Cette section a retenu l'attention des critiques (Berré \& Besse 2012, Caravolas 1994 et 2000, Stéfanini 1954 et 1994) qui signalent la clairvoyance de l'auteur et son influence posthume.

6. L'Essay est organisé en deux parties: la première comprend huit chapitres, consacrés à « l'explication des termes de la grammaire » (chapitre 1) aux parties du discours (chapitres 2 à 7), et à la syntaxe (chapitre huitième, "De la syntaxe et du style ", 130-155), et une section non numérotée «Adjonction particulière pour les Flamands" (156-164); la deuxième partie est composée de trois traités, dont un important traité « De la prononciation et de l'orthographe » (165-232).

7. Sur l'analyse détaillée de cette section, $c f$, Berré \& Pagani-Naudet (2018).

8. Il s'agit de l'Inleydinghe tot de francoysche taele, parue à Anvers en 1659 chez Jacques van Meurs.

9. Pour une présentation plus détaillée de l'organisation matérielle du livre, $c f$. Pagani-Naudet (2013).

10. Les exercices se donnent en effet par écrit : "afin que le disciple le copie, \& que tout d'un chemin il apprenne l'Orthographe de la langue » (Chiflet $1659: 152$ ).

11. Jean Antoine Caravolas consacre plusieurs pages de sa Didactique des langues à « la méthode de Chiflet » (1994 : 331). Il distingue trois étapes : « l'apprentissage des rudiments », « la lecture des auteurs », « l'expression orale ». Il nous semble que l'expression orale n'est pas une étape, mais une activité permanente de l'élève.

12. Caravolas observe que Chiflet qui « à propos de l'enseignement des débutants ne ménageait pas les détails et les exemples, n'a pour la deuxième étape, consacrée à la lecture de 'bons autheurs' et le récit qu'en font les élèves que six petites phrases ». Cette manière est «typique à la plupart des auteurs de méthodes de langues. Ils s'épuisent à écrire la manière d'enseigner la langue aux débutants et il ne leur reste que des généralités à dire pour le niveau supérieur. Pourtant enseigner le bon usage de la langue ne présente pas moins de problèmes que faire apprendre les structures de la langue et le vocabulaire de base » (1984:332-332). Dans le cas de l'Essay, on peut justement considérer que l'apprenant qui a passé l'étape des rudiments avec son maître est ensuite capable d'aborder les détails et curiosités de la langue contenus dans les chapitres qui précèdent, notamment dans les sections relatives aux « Observations ", destinés à polir le style et à accéder au bel usage.

13. Dans l'Essay, une part importante de la syntaxe (notamment les faits de rection) a été abordée avant le chapitre qui lui est explicitement consacré.

14. «Donc en ces premiers jours, il y aura tousjours exercice de la lecture » (Chiflet $1659: 154$ ).

15. Jacques Bourquin (2003:11) voit ainsi dans l'Essay «la grammaire d'un praticien qui veut faciliter l'apprentissage du français et non expliciter les mécanismes de la pensée ».

16. Cf. Margolin \& Michel (1976).

17. «Cette tendance à réduire au minimum l'étude des règles et des paradigmes, pour passer au plus tôt à la lecture de bons textes, est typique de la pédagogie des humanistes pour l'enseignement du latin. D'après Erasme c'est en parlant et en lisant les meilleurs auteurs qu'on parviendra à posséder la langue. Les conceptions d'Erasme étaient partagées par tous les humanistes et les pédagogues du temps [...]. Nous avons là les principes qu'adoptent les jésuites pour l'enseignement des langues classiques et que suivent aussi les maitres de français » (Pellandra $1990: 75)$.

18. Le texte paraît dans un ensemble de quatre petits ouvrages destinés à l'apprentissage du latin: il contient en outre Les declinaisons des noms et verbes que doibvent scavoir entierement par coeur les enfans, La maniere de tourner les noms, Pronoms, Verbes tant actifs que passifs, Des huict parties d'oraison. 
19. «aussi sur le Nom ou Verbe qu'il fera decliner par jour a son enfant, il luy en proposera ung autre ou plusieurs, qui se declineront selon l'exemple, en luy disant tousjours ce qu'ils signifient » (Cordier 1546 : 166-167).

20. «Quoique les Jésuites soient ses ennemis déclarés, Comenius reconnaît leur contribution au progrès de la pédagogie et en particulier à l'enseignement des langues. De leur côté, les Jésuites n'hésitent pas à publier sa Janua Linguarum Reserata, ce que l'auteur signale avec fierté » (Caravolas $1984: 126)$.

21. «On ne laisse pas les élèves procéder par bonds. On commence toujours par le texte et on finit par la grammaire. Huitièmement, il est impossible de faire avancer la connaissance chez l'élève autrement que par étapes graduées (axiome XI). Mes manuels qui mènent aux auteurs sont donc gradués (voir chapitre XII, art. 16) et ils sont bâtis de façon à ce qu'il existe également une gradation de l'un à l'autre. Alors ceux qui veulent en faire usage sont mis en garde contre la tentation des bonds » (Comenius [1648] $2005: 286$ ).

22. La relation pédagogique est une relation vivante, nécessitant la présence conjointe de l'élève et du maître. Le maître est « un guide qui le garde de l'erreur, qui l'éloigne des égarements, un guide [ducem habere] qui le console lorsqu'il manque le barreau, qui le maintienne dans le chemin » (Comenius [1648] $2005: 162$ ).

23. "Sans exemples, sans règles, sans exercices, rien ne s'enseigne, ni ne s'apprend, du moins correctement " [sine exemplis, praeceptis, exercitiis, aut nihil docetur et discitur aut nihil recte] (Comenius [1648] $2005: 171)$.

24. Cf. Swiggers (2013) et Claes (2010).

25. Maître de langue et auteur de plusieurs ouvrages pratiques : une grammaire et des colloques parus chez Plantin en 1557, un vocabulaire flamen-françoys, des Conjugaisons flamen francoises. Ces dernières sont toujours imprimées à Anvers du temps de Chiflet : en 1655, l'ouvrage est réédité chez Martinus Verhulst.

26. Chiflet a lui-même collaboré à la révision du Calepinus, dictionnaire multilingue.

27. Ainsi la conjugaison de l'optatif présente une table permettant de combiner une série de phrases complètes du type : «Je parleroys volontiers a madame par amours; je diroys voluntiers un mot a Henry Baude» (Meurier $1557: 37 \mathrm{r}^{\circ}$ ), ou pour le conjonctif «Combien que je parle François, si suis je bourguignon pour la vie» (Meurier $1557: 39 \mathrm{r}^{\circ}$ ). On ne connaît qu'une seule édition de cette grammaire parue en 1557 chez Plantin et il est difficile de savoir si Chiflet l'a eue en main. L'accès aux livres est toutefois, pour Chiflet, facilité par les liens de sa propre famille avec l'officine plantinienne. Même s'il a lui-même fait appel à d'autres imprimeurs, ses frères avaient presque systématiquement recours aux services de la maison Plantin-Moretus, et entretenaient avec Balthasar Moretus une riche correspondance.

28. On peut par exemple citer pour la seule ville d'Anvers une édition en latin (Exercia spirtualia s. p. Ignatii Loyolae, Antverpiae, Joannem Meursius, 1635) et une traduction (Les Exercices spirituels de S. Ignace de Loyola, Anvers, Michel Cnobbaert, 1673), sortant d'officines auxquels Chiflet lui-même fera appel. Il n'est pas inintéressant au demeurant de comparer la mise en page (recours à des alinéas numérotés) adoptée dans certaines éditions avec celle de l'Essay. Nous citons les Exercices d'après ces deux éditions anversoises.

29. «Le problème historique des Exercices était représenté par le fait qu'ils constituaient un moyen d'accès à un ample éventail d'expériences religieuses orientées, au contact direct avec la divinité » (Mongini 2014 : 130). Sur ces ajustements (mettre « l'accent sur les aspects pénitentiels, la conversion au péché, la confession et la prédication ", limiter "l'accès aux Exercices à certaines catégories de personnes ») permettant de concilier Exercices spirituels et orthodoxie de la Contre-Réforme (cf. Mongini 2014).

30. Les puissants moyens de mettre son salut en asseurance par un parfait amendement de vie, par le R.P. Laurent Chifletius. Anvers : Cornille Woons, 1656 ; Les Exercices des devotions chrétiennes pour chaque 
jour de la semaine. Bruxelles : François Foppens, 1707. Pour une bibliographie complète $c f$. Vrégille 2011.

31. L'édition anversoise que nous citons les désigne quant à elle par le mot « remarques ».

32. Ces annotations sont renforcées par une « guide ou directoire pour ceux qui font faire les dits Exercices » dans l'édition parisienne des Vrais exercices spirituels du P. S. Ignace de Loyola (Paris : J. Fouet, 1628). La première définit l'exercice : «Par le mot, Exercices spirituels, on entend toutes les manières d'examiner sa conscience, de méditer, de contempler, de prier mentalement et vocalement, enfin de s'acquitter deüement de toutes les operations de l'esprit. Car tout ainsi que se promener, faire voyage et courir, sont des exercices corporels : aussi préparer l'âme à ôter ses affections déréglées, et ensuite à rechercher \& à reconnaître la volonté de Dieu sur le dessein de notre vie, \& le salut de notre ame, ce sont des Exercices Spirituels » (Loyola $1673: 31$ ).

33. «Not enim abundantia scientiae, sed sensus \& gusuts rerum interior desiderium animae explere solet » (Loyola $1635: 13$ ).

34. Le système de renvois internes n'est pas sans rappeler celui qui est à l'œuvre dans la grammaire de Chiflet. L'édition latine est tout aussi précise: «[...] tradi potest Particulare primum Examen infrà positum, pag. 24 deinde geneale pag. 27. simulque modus orandi manè par horae dimidium, ex praeceptorum Dei [...]» (Loyola 1635: 19). L'édition parisienne des Vrais Exercices en revanche reste plus allusive et renvoie au premier examen ci dessous.

35. Expérience au demeurant difficile à établir : dans sa biographie, on le voit enseigner dans les premières années la grammaire latine mais rien n'est signalé concernant une activité d'enseignement du français.

36. Pour Stéfanini, « la méthode du P. Chifflet dont la grammaire enseigna le français à la plupart des étrangers qui voulurent l'apprendre sous Louis XIV (apprentissage immédiat des temps les plus usités d'avoir et d'être et des quelques mots de vocabulaire qui mettent aussitôt l'élève en état de dire : j'ai faim, nous avons soif, je suis Anglais, vous êtes aimable...) est à peu près la nôtre, à l'heure des laboratoires de langue et de la linguistique appliquée » (1994: 18).

\section{RÉSUMÉS}

Dans l'Essay d'une parfaite grammaire, Chiflet donne des conseils sur la « manière d'enseigner et d'apprendre la langue française » $(1659: 151-155)$ à un étranger. Il envisage un apprentissage actif, exigeant de l'apprenant qu'il s'exerce, selon ses moyens, à chaque étape de son parcours, jusqu'à ce qu'il parvienne à l'autonomie linguistique. Cette approche doit beaucoup à la formation spirituelle de Laurent Chiflet. Prêtre, et membre de la compagnie de Jésus, il étend au français, discipline dont l'enseignement n'était pas encore défini et encadré par l'institution, son expérience de pédagogue et de directeur spirituel. La section sur « la manière d'enseigner et d'apprendre » illustre la continuité qui existe entre la pédagogie et la spiritualité jésuites, entre le Ratio studiorum et les Exercices spirituels. Relation au maître, relation au livre, mise en œuvre des capacités naturelles et humaines de l'apprenant : entre le parcours de l'élève et le cheminement du retraitant pratiquant les Exercices spirituels, les analogies sont nombreuses. Il s'agit dans cet article de les explorer et de voir jusqu'où l'on peut pousser la comparaison entre l'ascèse menant à la perfection spirituelle et le « chemin à la perfection de la langue ».

In the Essay d'une parfaite grammaire, Chiflet gives advices on teaching and learning the French language to a stranger (La manière d'apprendre et d'enseigner la langue française 1659: 151-155). He 
describes a method for an active learning, requiring the learner to exercise himself, according to his means, at each stage of his journey, until he achieves linguistic autonomy. This approach owes much to the spiritual formation of Laurent Chiflet, a priest, member of the Sociey of Jesus. The section on "Teaching and Learning" illustrates the continuity between Jesuit pedagogy and spirituality, between Ratio Studiorum and the Exercices spirituels. Relationship to the teacher, relationship to the book, implementation of the natural and human abilities of the learner: between the path of the pupil and the path of the retreatant practicing the Spiritual Exercises, the analogies are numerous. The purpose of this article is to explore them and to see to what extent the comparison can be made between asceticism leading to spiritual perfection and the "path to the perfection of language".

INDEX

Keywords : Chiflet, Loyola, grammar, handbook, exercise, spiritual exercises, Jesuit

Mots-clés : Chiflet, Loyola, grammaire, manuel, Flamands, exercice, exercices spirituels, jésuite

\section{AUTEUR}

\section{CENDRINE PAGANI-NAUDET}

Université Côte d'Azur (BCL, UMR7320) cendrine.pagani@gmail.com 